\title{
TAMANHO DE FRUTOS E DE SEMENTES E SUA INFLUÊNCIA NA GERMINAÇÃO DE JATOBÁ-DO-CERRADO (Hymenaea stigonocarpa var. stigonocarpa Mart. ex Hayne, LEGUMINOSAE - CAESALPINOIDEAE) ${ }^{1}$
}

\author{
SILVIA RAHE PEREIRA², GISSELI RAMALHO GIRALDELLI ${ }^{3,}$ VALDEMIR ANTÔNIO LAURA ${ }^{4}$, \\ ANDRÉA LÚCIA TEIXEIRA DE SOUZA ${ }^{5}$
}

\begin{abstract}
RESUMO - Neste estudo foi descrito a biometria de frutos e de sementes de uma população de Hymenaea stigonocarpa var. stigonocarpa foi avaliado o efeito da massa de sementes na proporção e no tempo de germinação, sob condições de laboratório (câmara de germinação) e em viveiro, considerando como critérios de germinação a protrusão da raiz primária e a expansão de eófilos. Esta população de jatobá-do-cerrado apresentou uma grande variação no tamanho de frutos e de sementes. A massa das sementes variou entre 0,546 e 7,666 g e estas foram classificadas em pequenas, médias e grandes. Uma proporção maior de sementes classificadas como médias, emitiu a raiz primária em relação às grandes, enquanto as pequenas ocuparam posição intermediária. A massa de sementes não influencia a proporção de sementes que apresenta expansão de eófilos. As sementes grandes emitem a raiz primária mais rapidamente que as pequenas. $\mathrm{O}$ tempo médio da expansão de eófilos decresce com a redução da massa de sementes. Apesar das sementes médias e pequenas possuírem maior potencial para expor a raiz primária, estas apresentam maior taxa de mortalidade na transição entre as fases de protrusão da raiz primária e de expansão de eófilos.
\end{abstract}

Termos para indexação: biometria, protrusão da raiz primária, expansão de eófilos.

FRUIT AND SEED SIZE AND THEIR INFLUENCE ON THE GERMINATION OF Hymenaea stigonocarpa var. stigonocarpa Mart. ex Hayne (LEGUMINOSAE- CAESALPINOIDEAE)

\begin{abstract}
This study describes the biometry of the fruits and seeds of a population of Hymenaea stigonocarpa var. stigonocarpa. The effect of seed weight on the proportion and time of germination was evaluated under laboratory (BOD incubator) and nursery conditions, considering as criteria for germination, primary root protusion and eophylum expansion. This population of H. stigonocarpa var. stigonocarpa varied significantly in fruit and seed size with seed weights ranging between 0.546 and $7.666 \mathrm{~g}$ and classified as small, medium and large. A higher proportion of medium-sized seeds emitted the primary root compared to large seeds, whereas small seeds showed an intermediate pattern. However, seed weight did not influence the proportion of seeds that expanded the eophylum. The primary root of large seeds emerged faster than those of small
\end{abstract}

${ }^{1}$ Submetido em 20/11/2009. Aceito para publicação em 16/08/2010.

${ }^{2}$ Bióloga, Doutoranda do Programa de Pós-Graduação em Ecologia e Recursos Naturais, Universidade Federal de São Carlos, CP 676, CEP 13565-905, São Carlos, SP. E-mail: silviarahe@gmail.com

${ }^{3}$ Bióloga, Ms., Secretaria de Meio Ambiente e Desenvolvimento Urbano da Prefeitura Municipal de Campo Grande, MS, Rua Mal Rondon, 2655, Centro, CEP 79002-943, Campo Grande, MS. E-mail: gigiraldelli@yahoo.com.br

${ }^{4}$ Eng. Agrônomo, Pesquisador da Embrapa Gado de Corte, CP 154, CEP 79002-970, Campo Grande, MS. E-mail: valdemir@cnpgc.embrapa.br

${ }^{5}$ Bióloga, Profa. Dra., Departamento de Hibrobiologia, CCBS, Universidade Federal de São Carlos, CP 676, CEP 13565-905, São Carlos, SP. E-mail: altdesouza@gmail.com 
ones. The mean time of eophylum expansion decreased with decreasing seed weight. The results showed that although medium and small seeds have a higher probability of producing the primary root, they also show a greater mortality rate in transition to the eophylum expansion stage.

Index terms: Biometry, primary root protusion, lophylum expansion

\section{INTRODUÇÃO}

É crescente o interesse na propagação de espécies florestais nativas, o que demanda informações básicas sobre a morfologia e características germinativas das sementes dessas espécies (Silva e Carvalho, 2008). Estudos envolvendo descrições morfo-fisiológicas de frutos e sementes são importantes na identificação de espécies e na utilização destas em projetos de restauração de áreas degradadas ou mesmo para fins silviculturais, uma vez que subsidiam a análise de testes de germinação em laboratório e o entendimento das estratégias reprodutivas das espécies estudadas (Baskin e Baskin, 1998; Oliveira et al., 2005). Além disto, a morfometria de frutos e de sementes é uma importante característica usada em estudos sobre o mecanismo de dispersão e em projetos de preservação de populações naturais (Farias e Davide, 1993). Os métodos adequados para análises de sementes de espécies florestais tem sido alvo de grande interesse, especialmente quando visa à obtenção de informações que expressem a qualidade fisiológica da semente (Cruz e Carvalho, 2003). No entanto, os dados disponíveis sobre a caracterização de frutos e sementes e as informações silviculturais com relação ao potencial germinativo ainda são bastante escassos para espécies tropicais nativas (Cruz et al., 2001a; Araújo Neto et al., 2003; Silva e Carvalho, 2008).

A massa das sementes influencia vários aspectos da ecologia das espécies vegetais, uma vez que pode refletir as relações entre plantas e os agentes dispersores e predadores de sementes e as taxas de sobrevivência dos indivíduos na fase de plântulas (Westoby, 1998). Vários estudos avaliaram o efeito do tamanho de sementes, expresso através de sua massa, na germinação e estabelecimento de plântulas e comparando diferentes espécies vegetais (Geritz et al., 1999; Coomes e Grubb, 2003; Silvertown e Bullock, 2003), no entanto, existem ainda poucas informações disponíveis destes processos dentro de uma mesma espécie, especialmente em ambientes tropicais (Alves et al., 2005). A hipótese da relação positiva entre a massa de sementes e seu potencial germinativo sugere que sementes maiores possuam embriões bem mais desenvolvidos e uma maior quantidade de reservas (Bezerra et al., 2002), configurando-lhes uma maior qualidade fisiológica (White e González, 1990; Bezerra et al., 2004). Isto poderia aumentar as suas chances de sobrevivência sob condições de estresse hídrico, escassez de nutrientes no solo e condições desfavoráveis de intensidade luminosa (White e González, 1990; Silva e Carvalho, 2008).

A germinação é um fenômeno fisiológico evidenciado pela emergência da raiz primária, sendo que o desenvolvimento subseqüente é considerado como pósgerminativo (Bewley e Black, 1994; Nassif et al., 1998). No entanto, para estudos ecológicos, silviculturais e de tecnologia de sementes que visam a produção de mudas, a emergência da raiz primária não constitui indício capaz de prever o estabelecimento da plântula (Ferraz et al., 1998) uma vez que, somente a emergência e o desenvolvimento das estruturas essenciais do embrião manifestariam a sua capacidade de originar uma plântula normal, sob condições ambientais favoráveis (Nassif et al., 1998). Nestes casos, a semente é considerada germinada somente quando há a expansão dos eófilos.

Hymenaea stigonocarpa var. stigonocarpa Mart. ex Hayne (Leguminosae: Caesalpinoideae), popularmente conhecida como jatobá-do-cerrado, é uma espécie arbórea, heliófila, de até $12 \mathrm{~m}$ de altura e se distribui nas regiões sazonais, no cerradão e no cerrado sensu stricto do centro e sudeste do Brasil (Ratter et al., 1996). Floresce entre os meses de dezembro e fevereiro e frutifica entre setembro e novembro, apresentando frutos com formato aproximadamente cilíndrico a oblongo (Lee e Langenheim, 1975). Suas sementes são grandes, seu crescimento em campo é lento e sua madeira é de alta densidade (aproximadamente 0,90 g. $\mathrm{cm}^{-3}$ ) (Carvalho, 2007), o que a caracteriza como espécie secundária tardia. 
É capaz de colonizar áreas de pasto abandonado e é indicada para plantios em projetos de recuperação de áreas degradadas (Barbosa e Martins, 2003).

Este estudo caracterizou as dimensões dos frutos e a distribuição de frequência da massa de sementes de uma população de H. stigonocarpa var. stigonocarpa, localizada ao norte do Estado do Mato Grosso do Sul, e visou avaliar o efeito do tamanho da semente (pequena, média e grande) em duas fases do processo de germinação: a protrusão da raiz primária e a expansão de eófilos.

\section{MATERIAL E MÉTODOS}

A coleta de frutos de H. stigonocarpa var. stigonocarpa Mart. ex Hayne (Leguminosae: Caesalpinoideae), foi realizada em outubro de 2004, em árvores isoladas em áreas de pastagem da Fazenda Diamante, localizada no Distrito de Jauru, Município de Coxim, na região norte do Estado de Mato Grosso do Sul (18 41' $29^{\prime}$ 'S e $54^{\circ} 24^{\prime} 39^{\prime}$ 'W). A área possui 900 ha, sendo composta por pastagem e manchas de vegetação nativa de capoeiras, cerrado sensu stricto, mata de encosta e mata ciliar. O clima é do tipo tropical quente e sub-úmido, com quatro meses de seca, de junho a setembro.

Os dados de biometria foram obtidos a partir de 490 frutos coletados de dez árvores distanciadas entre si de pelo menos $100 \mathrm{~m}$ para aumentar a representatividade genética da população. Todos os frutos foram medidos quanto ao comprimento máximo, largura e espessura com paquímetro digital de precisão de $0,01 \mathrm{~mm}$. Posteriormente, foi efetuado o beneficiamento utilizando-se martelo para abertura dos frutos, uma faca para a retirada do excesso de polpa e esponja úmida para limpeza do resíduo de polpa nas sementes. Todas as sementes contidas nos frutos $(\mathrm{N}=$ 1315), que não apresentavam danos causados por insetos, fungos e outros patógenos, tiveram sua massa registrada, utilizando balança com precisão de $0,001 \mathrm{~g}$.

Entre os meses de abril e agosto de 2006, foi conduzido um experimento para avaliar o efeito da massa de sementes na proporção e no tempo médio de germinação (dias), utilizando como critérios a protrusão da raiz primária e a expansão de eófilos. As sementes intactas foram classificadas em três categorias: grandes $(>$ $5,0 \mathrm{~g}$ ), médias (entre 3,5 e $4,5 \mathrm{~g})$ e pequenas $(<3,0 \mathrm{~g})$. As categorias pequena e grande foram baseadas no primeiro e terceiro quartis da curva de distribuição de freqüências de massa. As sementes cuja massa era eqüidistante entre grandes e pequenas foram consideradas como médias. As sementes dos demais tamanhos foram descartadas a fim de evitar que valores marginais próximos aos limites das classes influenciassem os resultados.

Para o teste de germinação foram utilizadas 120 sementes de cada categoria de massa, previamente escarificadas com lixa d'água (№ 80) e colocadas em placas de Petri ( $150 \mathrm{~cm}$ de diâmetro) contendo vermiculita. As placas foram divididas em quatro repetições de 30 sementes, de acordo com cada categoria de massa, e foram colocadas em câmara de germinação (BOD), sob a temperatura constante de $30{ }^{\circ} \mathrm{C}$. O experimento foi avaliado diariamente por 60 dias até que todas as sementes tivessem apresentado a emissão da raiz primária e as remanescentes estivessem em fase de decomposição. As sementes pertencentes a categoria de massa que emitiram a raiz primária com o comprimento superior a $2 \mathrm{~mm}$ foram registradas e transplantadas para tubetes contendo substrato comercial (Plantmax $\left.{ }^{\circledR}\right)$. Os tubetes foram mantidos em casa de vegetação e umedecidos duas vezes ao dia. O experimento, nesta etapa em casa de vegetação, passou a ser monitorado semanalmente e todas as plântulas que apresentaram a expansão de eófilos foram registradas.

Os dados obtidos das variáveis, proporção de sementes germinadas e tempo médio gasto na fase de emissão da raiz primária e na fase expansão de eófilos foram avaliados mediante a análise de variância e, em caso de significância, as médias foram comparadas pelo teste te Tukey em 5\% de probabilidade. Os dados de proporção de sementes germinadas foram transformados em arcsen

$\sqrt{x / 100}$, sendo $\mathrm{x}$ a porcentagem de sementes com emissão da raiz primária ou de expansão dos eófilos.

\section{RESULTADOS E DISCUSSÃO}

Uma grande variação no tamanho de frutos foi registrada para a população de $H$. stigonocarpa var. stigonocarpa (Tabela 1). O comprimento dos frutos variou em 4,24 vezes, a largura variou em 6,06 vezes e a espessura variou em 2,88 vezes. A maior parte dos frutos $(62,6 \%)$ apresentou entre 105 e $155 \mathrm{~mm}$ de comprimento. O número de sementes variou entre um e 15 e uma grande proporção dos frutos $(42,3 \%)$ continha três ou quatro sementes (Figura 1). A massa de sementes variou de 0,546 a 7,666 g, uma variação equivalente a aproximadamente 14,04 vezes (Figura 2). 
TABELA 1. Dimensões ortogonais (comprimento, largura e espessura) de frutos de Hymenaea stigonocarpa var. stigonocarpa. ( $\mathrm{N}=$ número de frutos, $\mathrm{EP}=$ erro padrão, $\mathrm{CV}=$ coeficiente de variação)

\begin{tabular}{lccccc}
\hline Dimensões $(\mathrm{mm})$ & $\mathrm{N}$ & Média $\pm \mathrm{EP}$ & Mínima & Máxima & CV $(\%)$ \\
\hline Comprimento & 489 & $136,23 \pm 1,44$ & 57,99 & 246,00 & 23,4 \\
Largura & 489 & $48,32 \pm 0,39$ & 12,57 & 76,20 & 17,8 \\
Espessura & 489 & $34,68 \pm 0,21$ & 17,04 & 49,10 & 13,6 \\
\hline
\end{tabular}

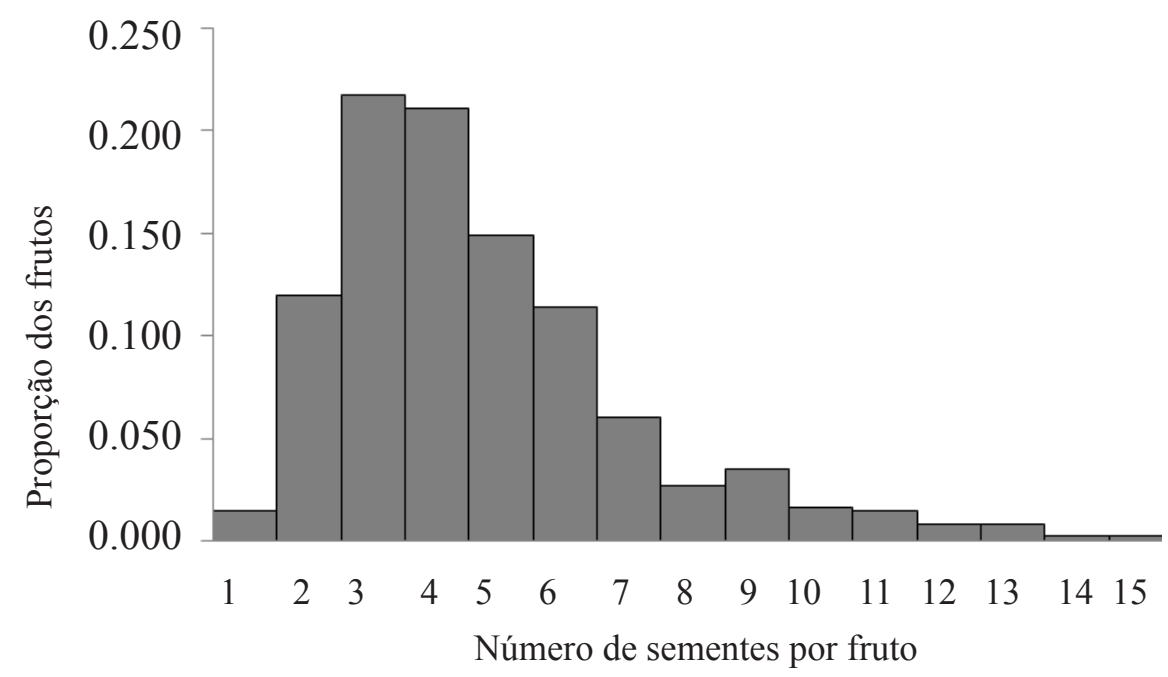

FIGURA 1. Distribuição da proporção de frutos de Hymenaea stigonocarpa var. stigonocarpa em relação ao número de sementes por fruto.

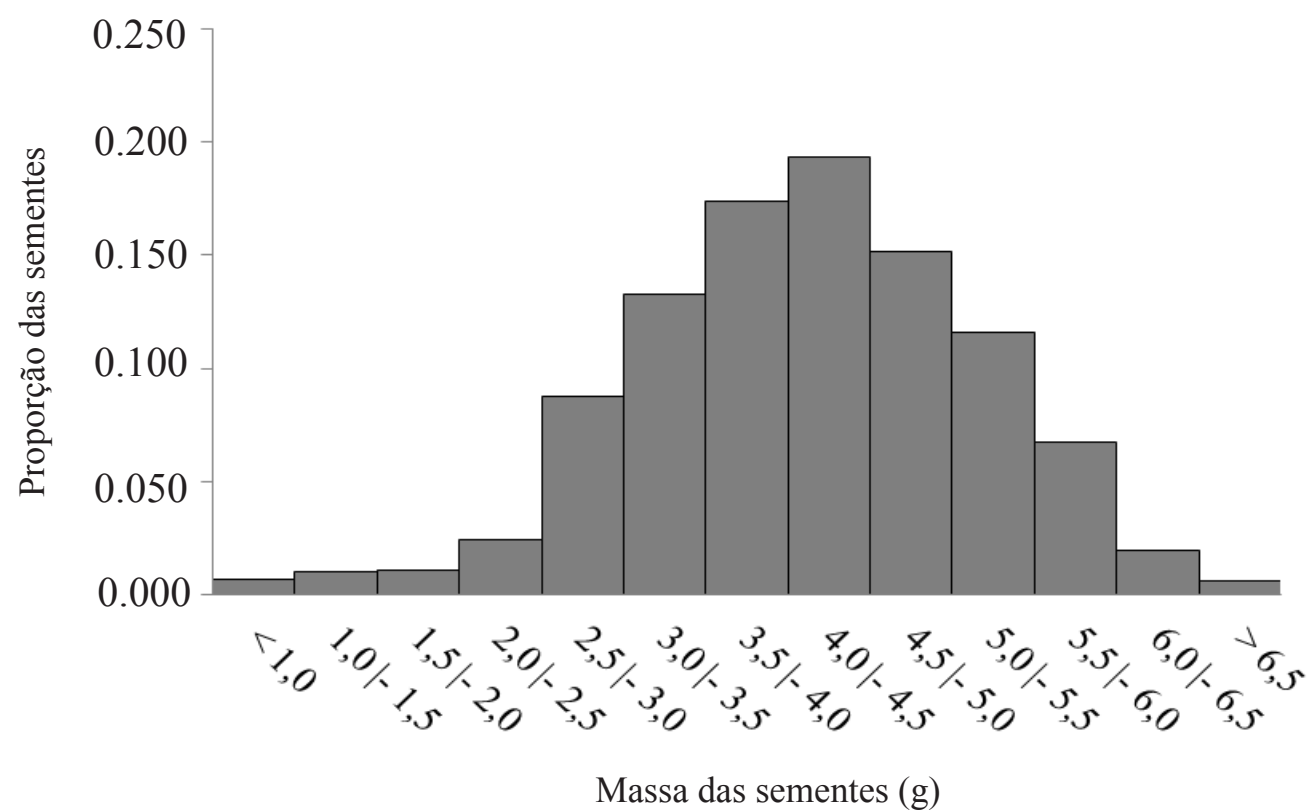

FIGURA 2. Distribuição da proporção de sementes de Hymenaea stigonocarpa var. stigonocarpa em diferentes classes de massa. 
As espécies arbóreas tropicais apresentam grande variabilidade com relação ao tamanho dos frutos, número de sementes por frutos e massa de sementes (Cruz et al., 2001b; Cruz e Carvalho, 2003; Gusmão et al., 2006). A descrição biométrica constitui um instrumento importante para detectar a variabilidade genética dentro de populações de uma mesma espécie e pode fornecer informações importantes para a diferenciação de espécies do mesmo gênero (Cruz e Carvalho, 2003; Gusmão et al., 2006). Frutos de $H$. courbaril são em média cerca de quatro vezes maiores que os frutos de $H$. intermedia (Cruz et al., 2001b) que por sua vez também possui frutos e sementes menores do que de $H$. stigonocarpa var. stigonocarpa, a espécie deste estudo. Além disto, H. intermedia também possui menor variação no número de sementes por fruto (1 a 3 ) e massa de sementes $(3,646$ a 5,300 g) do que a encontrada para $H$. stigonocarpa var. stigonocarpa.

Após 60 dias do início do experimento, 311 sementes apresentaram a emissão da raiz primária, representando $86,4 \%$ do total de sementes utilizadas. A massa de sementes influenciou significativamente a primeira fase do processo de formação de plântulas, tanto na proporção de sementes que expuseram a raiz primária quanto no tempo médio gasto nesta fase (Tabela 2). A proporção de sementes grandes que superaram esta fase foi menor do que as sementes médias, e as sementes pequenas ocuparam uma posição intermediária. Por outro lado, as sementes grandes expuseram a raiz primária mais rapidamente que sementes pequenas e, neste caso, as médias ocuparam posição intermediária. No entanto, a massa das sementes não influenciou a proporção de sementes que completaram todo o processo de germinação até a expansão dos eófilos em relação ao número inicial de sementes no experimento. O tempo médio observado para a expansão de eófilos decresceu com a redução da massa de sementes.

TABELA 2. Proporção e o tempo médio (dias) de germinação de indivíduos de Hymenaea stigonocarpa var. stigonocarpa considerando como critérios de germinação a emissão da raiz primária e expansão de eófilos. (Média \pm erro padrão)

\begin{tabular}{|c|c|c|c|c|}
\hline \multirow{2}{*}{$\begin{array}{l}\text { Categoria das } \\
\text { sementes }\end{array}$} & \multicolumn{2}{|c|}{ Proporção de indivíduos } & \multicolumn{2}{|c|}{ Tempo médio (Dias) } \\
\hline & $\begin{array}{l}\text { Emissão da raiz } \\
\text { primária }\end{array}$ & $\begin{array}{l}\text { Expansão dos } \\
\text { eófilos }\end{array}$ & $\begin{array}{l}\text { Emissão da raiz } \\
\text { primária }\end{array}$ & $\begin{array}{l}\text { Expansão } \\
\text { dos eófilos }\end{array}$ \\
\hline Pequenas & $0,892 \pm 0,034 \mathrm{ab}$ & $0,825 \pm 0,055$ & $8,51 \pm 0,29 \mathrm{a}$ & $19,10 \pm 0,25 \mathrm{c}$ \\
\hline Médias & $0,950 \pm 0,031 \mathrm{a}$ & $0,867 \pm 0,024$ & $8,17 \pm 0,15 \mathrm{ab}$ & $21,03 \pm 0,92^{b}$ \\
\hline Grandes & $0,750 \pm 0,051 \mathrm{~b}$ & $0,725 \pm 0,052$ & $6,89 \pm 0,06 b$ & $24,92 \pm 1,09^{a}$ \\
\hline$F_{2,9}$ & $8,936^{* *}$ & $2,315^{\mathrm{ns}}$ & $4,878^{*}$ & $50,236^{* * *}$ \\
\hline
\end{tabular}

As médias dentro de cada coluna seguidas da mesma letra não diferem entre si pelo teste de Tukey. ns - Não significativo; $* \mathrm{P}<0,05 ; * * \mathrm{P}<0,01$ e $* * * \mathrm{P}<0,001$

A separação das sementes por classes de massa, usada na determinação da qualidade fisiológica, mediante os testes de germinação e de vigor, tem sido frequentemente citada na literatura visando determinar uma classe de tamanho que maximize o rendimento na produção de mudas viáveis de diversas espécies vegetais (Torres, 1994; Malavasi e Malavasi, 2001; Macedo et al., 2009). Alguns estudos mostram que a massa da semente influencia as taxas de germinação e o vigor de plantas jovens, dentro de uma mesma espécie (Simons e Johnston, 2000; Bezerra et al., 2004; Costa et al., 2006; Klein et al., 2007). Por outro lado, há aqueles onde não é encontrada uma relação significativa entre a massa de sementes e a germinação (Bezerra et al., 2002; Oliveira et al., 2005; Duarte et al., 2006). Assim, a influência da massa de sementes na germinação e no vigor de plântulas parece ser específica, variando entre espécies e entre regiões distintas.

Muitos estudos que avaliam a porcentagem de germinação consideram como sementes germinadas aquelas em que é detectada a emissão da raiz primária (Alves et al., 2005; Limas et al., 2007; Varela et al., 2005), enquanto outros consideram germinadas apenas aquelas em que ocorre também a expansão de eófilos (Bezerra et al., 2002; Bezerra et al., 2004; Oliveira et al., 2005; Costa et al., 2006; Klein et al., 2007; Silva e Carvalho, 2008; Macedo et al., 2009). No entanto, não são registrados estudos na literatura que avaliem ambas as variáveis simultaneamente como no presente estudo, 
o que pode revelar mais detalhes sobre o processo de estabelecimento de novas plantas. Este estudo mostrou que a massa da semente pode influenciar o processo de germinação de $H$. stigonocarpa var. stigonocarpa quando se considera apenas a emissão da raiz primária, mas este efeito não foi detectado após a fase de expansão dos eófilos. É possível que sementes pequenas e médias possuam um maior risco de mortalidade durante a fase de transição entre emissão da raiz primária e a expansão de eófilos, quando comparadas com sementes grandes. Assim, se a germinação fosse considerada, adotandose como critério apenas a emissão da raiz primária, os resultados indicariam que sementes pequenas e médias possuem maior potencial germinativo, ao passo que se fosse considerada a expansão de eófilos como parâmetro de germinação, os resultados sugeririam que o tamanho de semente desta espécie não influencia a proporção de plântulas formadas.

Este estudo mostrou também que as sementes grandes de $H$. stigonocarpa var. stigonocarpa, apresentaram emissão da raiz primária num menor tempo, porém, levaram mais tempo para a expansão dos eófilos do que as sementes pequenas. $O$ efeito da massa da semente na velocidade de germinação parece variar muito entre espécies de diferentes hábitos e regiões geográficas (Baskin e Baskin, 1998). Teketay e Granstrom (1997), num estudo comparativo de 25 espécies da zona seca da Etiópia, mostraram que, em laboratório, a velocidade da germinação foi negativamente correlacionada com o tamanho da semente. Por outro lado, Paz et al. (1999) não encontraram efeito significativo entre o tamanho da semente e a taxa nem a velocidade de emergência de plântulas de sete espécies de Psychotria (Rubiaceae). A disponibilidade de água pode influenciar a relação entre tamanho de semente e as taxas e velocidades de germinação e, assim, os resultados de experimentos em campo e em casa de vegetação podem diferir entre si. Com o aumento do tamanho da semente, a relação entre a superfície e o volume da semente é reduzida, resultando na diminuição da capacidade de obtenção de água suficiente para iniciar o processo de germinação em solos secos (Harper e Benton, 1966).

\section{CONCLUSÕES}

Hymenaea stigonocarpa var. stigonocarpa apresenta grande variação no tamanho de frutos e na massa de sementes.
Sementes médias (entre 3,5 e 4,5 g) apresentam maior capacidade de emissão da raiz primária, que sementes grandes $(>5,0 \mathrm{~g})$.

Não há diferenças na proporção de sementes pequenas, médias e grandes que alcançam a fase de expansão dos eófilos.

$\mathrm{O}$ efeito da massa de semente na germinação de $H$. stigonocarpa var. stigonocarpa é dependente do critério usado para se quantificar a proporção de germinação de sementes: emissão da raiz primária ou expansão dos eófilos.

As sementes pequenas requerem um tempo maior para emissão da raiz primária, porém, um tempo menor para abrir e expandir os e ófilos quando comparadas às sementes grandes.

\section{REFERÊNCIAS}

ALVES, E.U.; BRUNO, R.L.A.; OLIVEIRA, A.P.; ALVES, A.U.; ALVES, A.U.; PAULA, R.C. Influência do tamanho e da procedência de sementes de Mimosa caesalpiniifolia Benth. sobre a germinação e vigor. Revista Árvore, v.29, n.6, p.877-885, 2005.

ARAÚJO NETO, J.C.; AGUIAR, I.B.; FERREIRA, V.M. Efeito da temperatura e da luz na germinação de sementes de Acacia polyphylla DC. Revista Brasileira de Botânica, v.26, n.2, p.249-256, 2003.

BARBOSA, L.M.; MARTINS, S.E. Diversificando o reflorestamento no estado de São Paulo: espécies disponíveis por região e ecossistema. São Paulo, SP: Instituto de Botânica, 2003. 63p.

BASKIN, C.C.; BASKIN, J.M. Seeds: ecology, biogeography, and evolution of dormancy and germination. London: Academic Press, 1998. 666p.

BEWLEY, J.D.; BLACK, M. Seeds: physiology of development and germination. New York: Plenum Press, 1994. 445p.

BEZERRA, A.M.E.; MEDEIROS FILHO, S.; MOREIRA, M.G.; MOREIRA, F.J.C.; ALVES, T.T.L. Germinação e desenvolvimento de plântulas de copaíba em função do tamanho e da imersão da semente em ácido sulfúrico. Revista Ciência Agronômica, v.33, n.2, p.5-12, 2002.

BEZERRA, A.M.E.; MOMENTÉ, V.G.; MEDEIROS FILHO, S. Germinação de sementes e desenvolvimento de plântulas de moringa (Moringa oleifera Lam.) em função do peso da semente e do tipo de substrato. Horticultura Brasileira, v.22, n.2, p.295-299, 2004. 
CARVALHO, P.E. Jatobá-do-Cerrado - Hymenaea stigonocarpa. Colombo: Embrapa Floresta, 2007. 8p. (Embrapa Floresta. Circular Técnica, n. 133).

COOMES, D.A.; GRUBB, P.J. Colonization, tolerance, competition and seed-size variation within functional groups. Trends in Ecology and Evolution, v.18, n.6, p.283-291, 2003.

COSTA, R.S.; OLIVEIRA, I.V.M.; MÔRO, F.V.; MARTINS, A.B.G. Aspectos morfológicos e influência do tamanho da semente na germinação do jambo-vermelho. Revista Brasileira de Fruticultura, v.28, n.1, p.117-120, 2006.

CRUZ, E.D.; CARVALHO, J.E.U.; LEÃO, N.V.M. Métodos para superação da dormência e biometria de frutos e sementes de Parkia nitida Miquel. (Leguminosae - Mimosoideae). Acta Amazônica, v.31, n.2, p167-177, 2001a.

CRUZ, E.D.; MARTINS, F.O.; CARVALHO, J.E.U. Biometria de frutos e sementes de jatobá-curuba (Hymenaea intermedia Ducke, Leguminosae - Caesalpinioideae). Revista Brasileira de Botânica, v.24, n.2, p.161-165, 2001b.

CRUZ, E.D.; CARVALHO, J.E.U. Biometria de frutos e germinação de sementes de Couratari stellata A. C. Smith (Lecythidaceae). Acta Amazônica, v.33, n.3, p.381-388, 2003.

DUARTE, E.F.; NAVES, R.V.; BORGES, J.D.; GIMARÃES, N.N.R. Germinação e vigor de sementes de cagaita (Eugenia dysenterica Mart. ex DC.) em função de seu tamanho e tipo de coleta. Pesquisa Agropecuária Tropical, n.36, v.3, p.173-179, 2006.

FARIAS, M.R.; DAVIDE, A.C. Aspecto morfológico do fruto, semente e plântulas de quatro espécies florestais nativas. Informativo Abrates, v.3, n.3, p.113, 1993.

FERRAZ, I.D.K.; VARELA, V.P.; MIRANDA, P.R.M.; CORRÊA, Y.M.B.; CARNEIRO, N.B. Efeito da temperatura na germinação de sementes florestais da Amazônia. In: HIGUCHI, N.; CAMPOS, M.A.A.; SAMPAIO, P.T.B.; SANTOS, J. (Ed.). Pesquisas florestais para a conservação da floresta e reabilitação de áreas degradadas da Amazônia. Manaus: INPA, 1998. p.185199.

GERITZ, S.A.; VAN DER MEIJDEN, E.; METZ, J.A.J. Evolutionary dynamics of seed size and seedling competitive ability. Theoretical Population Biology, v.55, n.3, p.324-343, 1999.

GUSMÃO, E.; VIEIRA, F.A.; FONSECA-JUNIOR, E.M.
Biometria de frutos e endocarpos de murici (Byrsonima verbascifolia Rich. ex A. Juss.). Cerne, v.12, n.1, p.84-91, 2006.

HARPER, J. L.; BENTON, R. A. The behaviour of seeds in soil: II. The germination of seeds on the surface of a water supplying substrate. Journal of Ecology, v.54, n.1, p.151-166, 1966.

KLEIN, J.; ZUCARELI, V.; KESTRING, D.; CAMILLI, L.; RODRIGUES, J.D. Efeito do tamanho da semente na emergência e desenvolvimento inicial de mudas de pitangueira (Eugenia uniflora L.). Revista Brasileira de Biociências, v.5, n.2, p.861-863, 2007.

LEE, Y.T.; LANGENHEIM, J.H. A systematic revision of the genus Hymenaea (Leguminosae; Caesalpinioideae; Detarieae). University of California Publications in Botany, v.69, p.1-109, 1975.

LIMAS, J.D.; SILVA, B.M.S.; MORAES, W.S. Germinação e armazenamento de sementes de Virola surinamensis (Rol.) Warb. (Myristicaceae). Revista Árvore, v.31, n.1, p.37-42, 2007.

MACEDO, M.C.; SACALON, S.P.Q.; SARI, A.P.; SACALON-FILHO, H.; ROSA, Y.B.C.J.; ROBAINA, A.D. Biometria de frutos e sementes e germinação de Magonia pubescens St.Hil (Sapindaceae). Revista Brasileira de Sementes, v.31, n.2, p.202-211, 2009.

MALAVASI, U.C.; MALAVASI, M.M. Influência do tamanho e do peso da semente na germinação e no estabelecimento de espécies de diferentes estágios da sucessão vegetal. Floresta e Ambiente, v.8, n.1, p.211215, 2001.

NASSIF, S.M.N.; VIEIRA, I.G.; FERNADES, G.D. Fatores externos (ambientais) que influenciam na germinação de sementes. Informativo Sementes-IPEF, 1998.

OLIVEIRA, I.V.M.; COSTA, R.S.; ANDRADE, R.A.; MARTINS, A.B.G. Influência do tamanho da semente na emergência das plântulas de longan (Dimocarpos longan Lour) Revista Brasileira de Fruticultura, v.27, n.1, p.171-172, 2005.

PAZ, H.; MAZER, S.J.; MARTINEZ-RAMOS, M. Seed mass, seedling emergence, and environmental factors in seven rain forest Psychotria (Rubiaceae). Ecology, v.80, n.5, p.1594-1606, 1999.

RATTER, J.A.; BRIDGEWATER, S.; ATKINSON, R.; RIBEIRO, J.F. Analysis of the floristic composition of the Brazilian Cerrado vegetation II: comparison of the woody vegetation of 98 areas. Edinburgh Journal of Botany, v.53, n.2, p.153-180, 1996. 
SILVA, B.M.S.; CARVALHO, N.M. Efeitos do estresse hídrico sobre o desempenho germinativo da semente de faveira (Clitoria fairchildiana R.A. Howard. - Fabaceae) de diferentes tamanhos. Revista Brasileira de Sementes, v.30, n.1, p.55-65, 2008.

SILVERTOWN, J.; BULLOCK, J.M. Do seedlings in gaps interact? A field test of assumptions in ESS seed size models. Oikos, v.101, n.3, p.499-504, 2003.

SIMONS, A.M.; JOHNSTON, M.O. Variation in seed traits of Lobelia inflata (Campanulaceae): sources and fitness consequences. American Journal of Botany, v.87, n.1, p.124-132, 2000.

TEKETAY, D.; GRANSTROM, A. Germination ecology of forest species from the highlands of Ethiopia. Journal of Tropical Ecology, v.13, n.6, p.805-831, 1997.
TORRES, S.B. Influência do tamanho das sementes de Acacia gomifera no desenvolvimento das mudas. Agropecuária Catarinense, v.7, n.2, p.5, 1994.

VARELA, V.P.; COSTA, S.S.; RAMOS, M.B. Influência da temperatura e do substrato na germinação de sementes de itaubarana (Acosmium nitens (Vog.) Yakovlev) Leguminosae, Caesalpinoideae. Acta Amazonica, v.35, n.1, p.35-39, 2005.

WESTOBY, M. The relationship between local and regional diversity: comment. Ecology, v.79, n.5, p.18251827, 1998.

WHITE, J.W.; GONZÁLEZ, A. Characterization of the negative association between seed yield and seed size among genotypes of common bean. Field Crops Research, v.23, n.3, p.159-175, 1990. 